\title{
Erratum to: Population genetics of self-incompatibility and developing self-compatible genotypes in niger (Guizotia abyssinica)
}

\author{
Mulatu Geleta $\cdot$ Tomas Bryngelsson
}

Published online: 23 July 2010

(C) Springer Science+Business Media B.V. 2010

\section{Erratum to: Euphytica \\ DOI 10.1007/s10681-010-0184-1}

Due to unfortunate circumstances, errors were introduced in the above mentioned article. Amongst others, when the text should have mentioned SC it stated SI instead. The correct sections are published below and should be treated as definitive by the reader:

The first sentence of the section "Identifying and developing self-compatible niger genotypes" should have read:

"A total of 340 plants from 200 populations, collected from all regions where the crop is grown in Ethiopia, were investigated for SC."

Below Table 3 the sentence must read: "With respect to the five main populations used in this study (Group A in Table 1), each S-allele was found in at least three populations (Table 1).

The online version of the original article can be found under doi:10.1007/s10681-010-0184-1.

\section{Geleta $(\bowtie) \cdot$ T. Bryngelsson}

Department of Plant Breeding and Biotechnology,

Swedish University of Agricultural Sciences, Box 101,

23053 Alnarp, Sweden

e-mail: mulatu.geleta@1tj.slu.se

T. Bryngelsson

e-mail: tomas.bryngelsson@1tj.slu.se
In the "Discussion" section the text should have read: "This systematic determination of the S-alleles finally resulted in the identification of ten S-alleles among the 60 plants investigated."

"The ten S-alleles identified based on RCPs and the $\mathrm{SC}$ allele $\left(\mathrm{S}_{\mathrm{c}}\right)$ from the self-compatible genotypes together add up to a minimum of 11 alleles at the $\mathrm{S}$-locus in G. abyssinica. Although the total number of S-alleles in the whole species could not be predicted based on the results of our study, the similarity in percentage compatibility within and among populations and the presence of each S-allele in samples from at least three of the five populations suggest a limited number of S-alleles (probably <20) in G. abysinica."

"The best approach, however, is to introduce the newly identified $S_{c}$ allele into target populations."

The first paragraph of the section "Self-compatibility in G. abyssinica" must read as follows:

"The results of this study demonstrate that true SC is rare in G. abyssinica. Although some degree of selfed seedset in several plants/population is possible (Riley and Belayneh 1989; Nemomissa et al. 1999), this does not necessarily mean that such plants are truly self-compatible as they may not set seeds in the following generations. Nemomissa et al. (1999) also reported such occurrences in this species. This condition may be considered to be pseudo-SC, which is dictated by segregating polygenic modifiers of S-gene action (Takahashi 1973; Levin 1996). 
However, the fact that such plants become more and more self-incompatible in the following generations needs further investigation to confirm if this condition is in line with the general understanding of pseudo$\mathrm{SC}$. The rarity of true SC in niger is the major reason for the lack of self-compatible lines with desirable traits in the country where it is cultivated most, Ethiopia. Also, to the best of our knowledge, no self- compatible genotypes have been developed through the conventional selection breeding approach anywhere in the world. Therefore, the self-compatible lines identified and developed in this study are the first to be reported despite the long cultivation history of the crop. These self-compatible lines can be used both for breeding purposes and for developing mapping populations for genetic linkage analysis. 